\title{
Conflitos recentes, estruturas persistentes: notícias do Sudão ${ }^{1}$
}

\author{
Beatriz. Perrone-Moisés \\ Professora do Departamento de Antropologia - USP
}

\begin{abstract}
RESUMO: Em março de 2000, foi assinado no Sudão meridional um tratado de paz entre os Dinka e os Nuer. As formas da guerra civil que se estendia por décadas, bem como as soluções acordadas, descritas pela imprensa e nos próprios documentos, adquirem pleno sentido quando se consideram as penetrantes análises de Evans-Pritchard acerca dos Nuer. Persistentes ao longo das décadas, as estruturas nuer reveladas em suas obras passam pelo "teste da história", permitindo inclusive retomar suas contribuições à reflexão acerca da relação entre estrutura e história.
\end{abstract}

PALAVRAS-CHAVE: Nuer, Dinka, Sudão meridional, Evans-Pritchard, estrutura, história.

Num dia de março de 1999, um avião pousa num lugarejo chamado Wunlit, no Sudão meridional. Em terra, um homem alto e magro sorri e toma nos braços o líder dos visitantes, "seu inimigo". O recémchegado retribui o abraço, colocando sua mão sobre o coração do outro. Assim começa uma reportagem publicada pelo $W$ ashington Post, a 7 de julho de 1999, acerca de uma conferência de paz numa região africana tomada pela guerra havia anos. Os chefes que se abraçam são Madut Aguer Adel e Isaac Magok Galtuak. O primeiro é Dinka, o segundo, Nuer. Seu encontro marca o início de uma conferência que levaria a um acordo de paz ${ }^{2}$.

Quando Evans-Pritchard fez sua pesquisa entre os Nuer, entre 1930 e 1936, informava que eram aproximadamente 200 mil, vivendo ao sul da confluência do Nilo com o Sobat e o Bahr el Ghazal, na então colônia 
britânica do Sudão (1940: 3). Dizia, na mesma passagem, que sua semelhança com os vizinhos e inimigos Dinka era notável, que reconheciam ambos sua origem comum, e que não se conhecia "a história de sua divergência” (idem). Os Nuer continuam na mesma região, agora pertencente ao Sudão independente, e são hoje bem mais numerosos: em 1982, eram 740 mil no Sudão, e 40 mil na Etiópia. Em conjunto com os Dinka, cuja população é duas vezes maior ${ }^{3}$, constituem a esmagadora maioria no Sudão meridional, onde também se encontram outras etnias. Os acontecimentos destas últimas décadas mostram que as "divergências" entre Nuer e Dinka não só permanecem, como foram acirradas por conflitos mais amplos e pela introdução de armamento pesado. Mostram ainda que as "semelhanças" entre ambos, aquelas que Evans-Pritchard identificava como estruturais, persistem igualmente. Vejamos os fatos.

\section{A guerra civil e a resistência no Sudão Meridional}

Em 1956, o Sudão tornou-se independente do domínio britânico, e seu governo foi assumido por representantes da população muçulmana, majoritária no norte do país. Todas as etnias do sul começaram a reagir às autoridades muçulmanas ${ }^{4}$. Em 1983, o conflito agravou-se, quando o governo sudanês decidiu impor a lei islâmica a todo o país ${ }^{5}$. Criou-se então no sul o SPLA/M (Sudanese People's Liberation Army/Movement), que liderava a resistência armada ao governo. Embora o SPLA/M não contasse com o apoio de todas as etnias não-muçulmanas do Sudão meridional, visto ser composto de Dinka e Nuer, foi expandindo a guerrilha de resistência contra o governo durante a década de 1980, ganhando terreno no sul. Dinka e Nuer combatiam unidos, até que, em 1991, um oficial nuer tentou dar um golpe e tomar o lugar do comandante do SPLA/M, cargo ocupado por líderes dinka desde a criação do movimento. Nesse evento encontra-se um primeiro exemplo de aplicação direta das lições de Evans-Pritchard em Os Nuer a nossa leitura das 
Revista de Antropologia, São Paulo, USP, 2001, v. 44 nº 2.

notícias do Sudão. Evans-Pritchard (1940: 125) resume o mito que narra a origem da inimizade entre Nuer e Dinka: no início dos tempos, os Dinka roubaram gado dos Nuer, que por isso receberam do criador a incumbência perpétua de atacar os Dinka. Desde que essa situação de inimizade se instalou, conta Evans-Pritchard, "quase sempre" foram os Nuer a tomar a iniciativa dos ataques. Em 1991, não foi diferente. O golpe falhou, mas o oficial nuer recebeu apoio suficiente para que o movimento se cindisse, previsivelmente, ao longo da linha Dinka/Nuer. Iniciou-se assim uma outra guerra no sul, entre segmentos dinka e segmentos nuer, sobretudo ao longo da fronteira entre ambos, enquanto outros segmentos dinka e nuer continuavam combatendo o governo islâmico do norte, e outros ainda prosseguiam nas guerras intratribais. Não são "entidades étnicas" Nuer ou Dinka que entram em guerra, mas segmentos nuer e segmentos dinka, envolvidos em diversas guerras. A dinâmica segmentar que Evans-Prichard nos mostra como essencial entre os Nuer (e os Dinka) encontra-se aí em plena atividade. Sua importância reaparece claramente nas negociações que serão narradas mais adiante.

Certos contornos específicos da guerra entre os Dinka e os Nuer, observados por Evans-Pritchard, são também notáveis nessa guerra dos anos 90: tratava-se fundamentalmente de roubo de gado e rapto de pessoas. A introdução das armas de fogo e a inserção desse conflito numa guerra mais ampla produziu, contudo, modificações. A mesma reportagem do Washington Post conta que os próprios Dinka e Nuer reconheciam que havia poucas mortes em suas guerras antigas, mesmo porque a morte de um inimigo acarretava sérios perigos para o matador. Informa o artigo:

Tradition in both tribes held that causing a death created "spiritual pollution". A bit of the blood of any man a Nuer speared to death was thought to be in the slayer, and had to be bled out of the upper arm by an earth priest. To drink or eat before reaching the priest was to die $^{6} .(1999)$

Líderes rebeldes haviam argumentado, a certa altura, que tais perigos e suas decorrentes restrições não se aplicavam à morte de inimigos 
produzida por armas de fogo. Telar Deng, juiz dinka educado nos Estados Unidos, resumia: "They believe, The ghost of the deceased will not baunt me, because I did not kill with a spear?." "Once removed from its moral consequences, killing became easier", conclui o artigo. $\mathrm{O}$ fato é que todos os jovens dinka e nuer passaram a carregar cotidianamente armas pesadas, e as mortes se multiplicaram em proporções assustadoras. Ao longo dos anos 90, toda a faixa de fronteira Dinka/Nuer ficou vazia: as aldeias tinham sido dizimadas ou sua população tinha sido raptada e os eventuais sobreviventes haviam fugido. Não há como calcular quantas vítimas essa guerra civil no Sudão meridional fez. Estima-se que os conflitos iniciados há décadas, contra o norte muçulmano, nessa que é a mais longa guerra civil registrada no mundo, tenham feito algo como 2 milhões de vítimas, além de terem agravado a já endêmica situação de fome e epidemias na região?

\section{O tratado de paz de Wunlit}

Em junho de 1998, 35 chefes nuer e dinka e líderes religiosos se reuniram num local chamado Loki (Lokichogio, no Quênia) e iniciaram conversações pelo fim das hostilidades, do roubo de gado, dos raptos, das destruições de aldeias, pela restauração da possibilidade de transitar na fronteira dinka/nuer. O acordo prévio de Loki deu origem a uma conferência de paz Dinka-Nuer, em março de 1999, no vilarejo de Wunlit, em território dinka. Ali pousara o avião de que descia o chefe Isaac Magok, na primeira cena evocada acima. A presença em terras dinka de Magok, guerreiro respeitado e temido, era percebida pelos presentes como o indício mais forte de que o conflito acabaria.

Em Wunlit, pequena comunidade dinka, reuniram-se, pois, aproximadamente 1.300 pessoas: Dinka e Nuer, chefes, mulheres, jovens, velhos e crianças, líderes religiosos, líderes comunitários, chefes de pele-deleopardo nuer e "donos da lança" dinka, representantes do SPLM/A. 
A conferência foi aberta, no dia 27 de fevereiro de 1999, com o sacrifício de um Touro Branco (Mabior Thon [em dinka]/Tu Bor [em nuer]), sobre o sangue do qual pisaram os integrantes da conferência, simbolizando a vontade de superar as diferenças.

Em seguida, tratava-se de dar a cada parte a oportunidade de narrar sua versão dos fatos e apontar as questões que lhe pareciam fundamentais. Após três dias e meio de narrativas, questões e comentários, chegou-se ao estabelecimento dos itens a serem discutidos: 1. Desaparecidos e casamentos com pessoas raptadas; 2. Direitos à terra e restabelecimento de relações; 3. Arranjos institucionais; 4. Monitoramento das fronteiras; 5. Grupos não incluídos no processo de paz; 6. Extensão da paz à margem leste do Nilo e região equatorial. Grupos de trabalho reuniram-se, então, para discutir, em dinka e nuer exclusivamente, cada um dos itens, e apresentaram suas propostas, acordadas em consenso, após novas rodadas de propostas e contrapropostas.

O Acordo de Paz Dinka-Nuer de Wunlit foi assinado por 318 pessoas. Compreensível, tendo em vista que nem os Dinka nem os Nuer constituem unidades políticas centralizadas, mas pode-se imaginar que outros leitores dessas notícias se perguntem por que tantas assinaturas para selar um acordo entre dois "povos". Leitores de Os Nuer, percebemos ainda que tal acordo, embora intitulado "Conferência de Paz Dinka-Nuer", diz respeito à intenção de paz de apenas alguns segmentos nuer e dinka ${ }^{9}$. Entre as várias resoluções de Wunlit, encontram-se a reocupação de aldeias de fronteira abandonadas, a instalação de escolas mistas dinka/nuer na fronteira ${ }^{10}$, a promoção do bilingüismo (prêmios para bilingües dinka-nuer), a instalação de um centro veterinário comum, etc.

O encontro foi encerrado, a 8 de março, com "culto cristão e sacrifício tradicional", conforme narram todas as notas de imprensa relativas ao encontro de Wunlit. 


\section{Parentesco e casamento}

O item I do acordo de Wunlit, que diz respeito às pessoas desaparecidas e raptadas, contém várias disposições que remetem diretamente a formas culturais descritas por Evans-Pritchard, em Os Nuer, mas sobretudo na obra posterior, Kinship and Marriage among the Nuer. Seus subitens são:

A. Meninas raptadas ainda solteiras seriam devolvidas a suas famílias;

B. As meninas que tivessem se casado em cativeiro seriam consultadas quanto a desejarem voltar a seu local de origem ou permanecer: se quisessem voltar, teriam de ser liberadas imediatamente; se quisessem ficar, "then the bridewealth must be collected and presented to her parents/relatives"; se tivessem filhos desse casamento, "the natural father may choose to redeem the children according to Dinkal Nuer tradition"; "if the father refuses to redeem the children, the mother is free to leave with them";

C. As mulheres que já eram casadas anteriormente deviam ser devolvidas, com todos os seus filhos;

D. Todos os rapazes e homens raptados deveriam ser repatriados, a não ser que tivessem se casado e quisessem permancer onde estavam; se tivessem casado e quisessem voltar, "then bis family shall pay the bridewealth which was paid by his captor", e poderiam voltar com a mulher e os filhos.

Em primeiro lugar, o consentimento dos noivos, mencionado nos items B e D, corresponde a uma prática nuer verificada por EvansPritchard. Quanto ao significado dos outros itens, vejamos as informações de que dispomos. Os casamentos entre os Nuer eram feitos em várias "etapas", consistindo de dons e contradons entre os parentes do noivo e os da noiva, basicamente em gado. Para que um casamento fosse considerado válido, eram necessárias a união de fato e as prestações em gado que a reconfirmavam, não sendo nenhum dos dois suficiente (Evans-Pritchard, 1951: 58). Os casamentos entre mulheres raptadas e seus raptores não "existem”, portanto, a não ser que sejam devidamente confirmados por essas prestações, declarações e confirmações de laços entre as famílias. A quantidade de gado que assim passava entre as 
Revista de Antropologia, São Paulo, USP, 2001, v. 44 nº 2.

famílias era negociada, desde a "primeira prestação", em "conversas de gado" (riet ghok) (idem: 60). A efetivação de um casamento podia levar anos, até que o marido conseguisse entregar o número de cabeças combinado; antes disso, não havia casamento. $\mathrm{O}$ item $\mathrm{D}$ revela que o "preço da noiva”, indispensável para a primeira confirmação do casamento, havia sido pago pelos raptores, o que torna evidente que as regras se mantêm, e são reconhecidas de ambos os "lados" do conflito. Do mesmo modo que o casamento, a filiação (item B) só se torna legítima uma vez paga a prestação em gado (ruok), geralmente de 4 a 6 cabeças. O pagamento do preço da noiva em si não dá, ao marido, o direito legal sobre os filhos de sua esposa, mas sim o direito de legitimar cada um dos filhos desta, mediante o pagamento do ruok (idem: 118 s.s). Percebe-se (item C) que no caso de mulheres já casadas, não há possibilidade de quebrar o laço estabelecido pelo pagamento do preço da noiva ("devem ser devolvidas”), o que dá ao marido o "direito indiscutível" de tornar-se pater dos filhos de sua esposa, ainda que gerados por outro homem.

Todas essas relações de parentesco entre Nuer e Dinka, devidamente estabelecidas e reconhecidas, certamente reforçam a inserção de segmentos dos dois povos numa tessitura social comum. Já EvansPritchard notava que aproximadamente metade dos Nuer eram de ascendência dinka, incorporados principalmente por meio de adoção e casamento (1940: 221), de modo que não ocorre aqui algo de novo. Por outro lado, o "tecido" tradicional era, por natureza, "elástico", e o próprio Evans-Pritchard compara intercasamentos a "elastic bands" entre segmentos políticos (1940: 226): longe de criarem grandes unidades estáveis, as relações de parentesco que forneciam "the non-political texture of the political system" (1940: 228) permitiam afastamentos e aproximações, fissões e fusões sucessivas e constantes.

Com a assinatura da paz de Wunlit, em março de 1999, o processo de unificação política do Sudão meridional dava um passo considerado decisivo, por todos os participantes e observadores. Seis meses mais 
tarde, um Comunicado do Conselho de Paz Dinka-Nuer (Margem Ocidental $)^{11}$ relatava o andamento do processo. "Longas narrativas" e conversas entre representantes dos Dinka e dos Nuer, diz o documento, revelavam resultados animadores: 148 pessoas raptadas tinham retornado a seus lares e cinco "proper marriages" tinham sido realizados. Os relatórios dos grupos de trabalho apresentam detalhes. Dentre as pessoas raptadas, ainda havia oito dinka e 42 nuer desaparecidos. Os cinco casamentos em questão foram realizados entre os Nuer.

\section{Idioma bovino, tempo e espaço sociais}

O artigo citado bem como os textos das negociações do tratado de paz e relatórios subseqüentes são marcados por um "idioma bovino" (Evans-Pritchard, 1940: 19). Ecoando e confirmando as observações de Evans-Pritchard de que qualquer conversa com os Nuer acabava em gado - "they are always talking about their beasts" (idem) -, o artigo informa ainda a seus leitores de que uma das várias semelhanças entre os Dinka e os Nuer está em que "both honor their scrawny, hump-backed cattle as the center of the temporal world, at once wealth on the hoof and a mystical link to the spiritual plane". O gado não é, porém, objeto de nenhum dos itens do acordo de Wunlit, embora apareça em todos eles, de uma forma ou de outra, o que só faz confirmar seu estatuto de "linguagem", de "código" para expressar relações.

No Comunicado do Conselho de Paz citado acima, o "idioma bovino" tem o lugar privilegiado a que remetem essas informações. O primeiro resultado positivo do processo de paz relatado por esse documento é o retorno das pessoas raptadas, que mais adiante, nas estratégias propostas para a continuação dos esforços de paz, encontram-se reunidas com as cabeças de gado numa mesma "questão", bem como na recomendação de garantir serviços médicos "for human beings and cattle". O segundo é a devolução de 141 cabeças de gado. O detalhamento desse item informa: 
Revista de Antropologia, São Paulo, USP, 2001, v. 44 nº 2.

It was found that Nuer had not raided any Dinka cattle after the Wunlit Peace and Reconciliation Conference, but Dinka raided a total of 700 head of cattle from Nuer since the Wunlit Conference amnesty date of the $1^{\text {st }}$ of January, 1999. These raids occurred prior to Wunlit but were not covered by the amnesty.

Dessas setecentas cabeças de gado nuer, ainda faltavam, portanto, 559. Os autores dos ataques ("indivíduos de quatro áreas") são em seguida nomeados, e o número de cabeças devolvido por cada um especificado $^{12}$. Nas estratégias propostas para o prosseguimento do processo de paz, um item é dedicado ao modo sugerido para o recebimento do "preço da noiva". No Apêndice, as pessoas que se casaram são nomeadas e o número de cabeças pago para a efetivação dos casamentos especificados ${ }^{13}$.

O terceiro capítulo de Os Nuer, "Tempo e espaço", fornece as informações e análises que nos permitem apreciar ainda certos detalhes das negociações mencionados no artigo citado e nos documentos oficiais. Evans-Pritchard nos dizia que era na estação seca que os Nuer se reuniam em grupos mais numerosos, sendo então requerida "greater co-ordination and co-operative action" (1940: 103). Foi na seca que se realizaram todas as reuniões Dinka/Nuer. Entre as resoluções, uma das mais significativas é a de estabelecer um policiamento conjunto das áreas de pastagem (toich) e pesca durante a estação seca, a partir de então compartilhadas, em paz, por segmentos dos dois grupos ${ }^{14}$. A importância política de uma tal decisão pode ser avaliada quando se considera que, entre os Nuer descritos por Evans-Pritchard, pastagens eram compartilhadas por grupos próximos do ponto de vista da estrutura política. Esses detalhes das negociações confirmam que ações cooperativas são realizadas na estação seca, e a importância do fator ecológico, que por vezes é interpretada, por leitores de Os Nuer, como indício de uma concepção de "condicionamento" da cultura pelo meio, que não é a de Evans-Pritchard, ressurge como não negligenciável. Mais adiante, no mesmo capítulo, lê-se: "Great rivers flow through Nuerland and it is often these natural boundaries which indicate the lines of political cleavage" (1940: 112). 
Narrando os acontecimentos ligados à conferência de Wunlit, um observador comenta que a caminho da conferência, "the Nuer looked out the window of the plane, saw the crossing of 'their river' and knew they had entered Dinkaland for the first time" ${ }^{\text {"15. }}$. Fronteiras fluviais entre segmentos nuer e dinka também ficam claras no próprio título dos acordos e nos textos das negociações, que utilizam sempre a distinção entre "margem esquerda" e "margem direita". De fato, como observa Evans-Pritchard, os "Nuer attach values to their geographical distribution and these evaluations give us socio-spacial units" (: 190).

\section{Dinâmica segmentar}

Voltemos às negociações de paz. Celebrado entre segmentos Dinka e segmentos Nuer, o acordo de Wunlit afirmava (item IV) a necessidade de "estender a paz" à margem oriental do Nilo e à região equatorial, para que "todos possam viver em harmonia", e listava os conflitos a serem resolvidos na outra margem: Nuer-Dinka Bor; Murle-Nuer e Murle-Dinka Bor; Murle-Anyua; Nuer-Shilluk; Lou Nuer-Gaawar Nuer. Conforme aos mecanismos indicados pelo documento para atingir a paz, foram realizados, subseqüentemente, diversos "encontros de reconciliação" e "miniacordos de paz".

A 6 de novembro de 1999, Lou Nuer de diversas regiões se reuniram em Waat, onde declararam "an end to years of internal conflicts among our people, battles between different factions", ou, noutra formulação, "an end to all conflicts among Lou Nuer", em nome da paz que desejavam estendida aos Gawaar Nuer a oeste e aos Jikany Nuer a leste, e de modo mais amplo, a "todo o povo Nuer" e a todo o Sudão meridional. É notável, na formulação desse acordo, a afirmação das "unidades" Lou e Nuer, que, transformando guerras entre seções em conflitos "internos" entre "facções", parece negar a dinâmica segmentar que a própria existência do acordo, por outro lado, evidencia ${ }^{16}$. Outra conferência de paz, realizada em Liliir, em maio de 2000, reuniu “Anynak, Dinka (Bor \& 
Revista de Antropologia, São Paulo, USP, 2001, v. 44 nº 2.

Padang), Jie, Kachipo, Murle (Boma) and Nuer (Gawaar \& Lou)". Realizadas entre segmentos de outras populações do Sudão meridional, além de segmentos Dinka e Nuer, essas negociações e suas resoluções apresentam outro tipo de formulação, em que não há - e não surpreende - afirmação de unidade, mas sim afirmação de intenção de paz, em nome de um interesse comum, entre "unidades" reconhecidas como diferentes. Significativamente, o texto de uma das notas de imprensa relativas a esse encontro termina com um comentário em que se pode ler claramente a força da dinâmica segmentar:

The conference regretted however that the wishes of the Gawaar-Nuer to participate in the reconciliation process was denied by an Upper Nile faction. The delegates requested that these, and other groups who did not have opportunity to participate in the conference, be given a chance to meet and reconcile as soon as possible. This, they said, was the wish of the people. ${ }^{17}$

"We have moved from being enemies to brothers and sisters", afirmava o Comunicado do Conselho de Paz, "and now our unity strengthens the security for both Dinka and Nuer". Notemos, inicialmente, a formulação "brothers and sisters". Uma primeira leitura, considerando o fato de os acordos terem todos sido promovidos pelo Novo Conselho das Igrejas do Sudão, poderia sugerir a adoção de uma fórmula cristã. Porém, considerando a importância da linguagem do parentesco entre os Nuer, que "reckon all social ties in a kinship idiom" (Evans-Pritchard, 1940: 220), de modo que sua unidade (Os Nuer), inexistente no plano das relações políticas, se expressava nos mitos por uma única grande estrutura de parentesco (: 236), que as relações territoriais eram investidas de um "kinship value" (:231) e que os Nuer freqüentemente utilizavam, por delicadeza, termos de parentesco mais "próximos" do que aqueles que corresponderiam à relação de fato entre pessoas que se encontravam (Evans-Pritchard, 1940: 234), pode-se ler na formulação muito mais do que influência cristã, um "modo de falar" extremamente significativo entre os Nuer (talvez também entre os demais grupos envolvidos na negociação). Em suma, tratar-se-ia, justamente, de reafirmar, na lin- 
guagem privilegiada do parentesco, a unidade desejada entre os povos do Sudão meridional.

\section{Estruturas e história, estruturas históricas}

Considerando que o mecanismo de fissão-fusão, como mostra EvansPritchard em relação aos Nuer, é o princípio estrutural compartilhado por Dinka e Nuer, não nos espanta que os conflitos e as negociações de paz envolvam segmentos, capazes de se unir e se separar dependendo dos contextos políticos. Mas fusões entre Dinka e Nuer e, mais ainda, envolvendo outros povos do Sudão meridional, são novidades. E novidades cujos desdobramentos vale a pena acompanhar, sobretudo quando se pensa na afirmação de Evans-Pritchard, relativa aos processos de resolução de conflitos entre segmentos nuer, de que

Nuer know that "a feud never ends". There may be peace for a time [...] but enmity continues and the people on both sides remain jiter, people who are at feud, even if there is no overt hostility. There is no frequent fighting or continuous unabated hostility, but the sore rankles and the feud, though formally concluded, may at any time break out again. (: 155)

A citação se refere aos "feuds", inimizades entre segmentos relativamente próximos em termos estruturais, e distintos, pelos Nuer e por Evans-Pritchard, das "guerras", entre tribos. Entre segmentos de uma mesma tribo, a oposição, denominada ter, podia ser resolvida por meio de arbitragem e compensação, em gado ("blood-cattle"). Ainda assim, a inimizade persistia, indefinidamente. Do mesmo modo que os conflitos entre segmentos eram essenciais do ponto de vista da própria estrutura, pois reafirmavam continuamente a diferença entre os segmentos, a guerra, dizia Evans-Pritchard, era o que mantinha as relações estruturais entre as tribos Nuer e entre estes e outros povos (1940: 190). Os acordos comentados acima, embora revelem a persistência de mecanismos estruturais, teriam por objeto obter uma pacificação que, nesse sentido, afetaria as estruturas. 
Revista de Antropologia, São Paulo, USP, 2001, v. 44 nº 2.

Mas a história já tinha produzido "greater intertribal solidarity" entre os Nuer, como resposta à ofensiva "árabe-européia" (: 191). Em várias passagens de Os Nuer, Evans-Pritchard lembra que os conflitos com os árabes do norte já eram antigos. E, numa passagem específica (: 1859), analisa o processo histórico de unificação nuer, simbolizado pela emergência, então recente, dos profetas. Ao contrário dos chefes de pele-de-leopardo, "mechanisms of tribal structure", os profetas "were pivots of federation between adjacent tribes and personified the structural principle of opposition in its widest expression, the unit and homogeneity of Nuer against foreigners" (: 189). Essa passagem é, aliás, um belo exemplo de análise histórica por parte de um autor que, nisso também, revela seu modo peculiar de "pertencer" ao estrutural-funcionalismo britânico. Sabe-se o quanto as estruturas de Evans-Pritchard se afastavam da noção de estrutura social que prevalecia entre seus conterrâneos ${ }^{18}$. Menciona-se raramente a contribuição de Evans-Pritchard à análise antropológica da história, na obra pioneira The Sanusi of Cyrenaica ${ }^{19}$, bem como em diversas passagens de sua obra, como essa sobre a emergência dos profetas entre os Nuer. Evans-Pritchard resume essa análise assim:

Certain structural changes were taking place in response to changed conditions: the development of functions that were more purely political than any exercised by individuals before and of a greater degree of unity among neighbouring tribes than there had been hitherto. (: 189)

$\mathrm{Na}$ ausência de uma análise aprofundada, não nos é possível afirmar que os chefes mencionados no início, por exemplo, ocupem estruturalmente esse papel centralizador que cabia aos profetas. Mas as brilhantes análises de Evans-Pritchard permitem-nos ver que as mudanças são, de fato, estruturais. Numa conferência de 1961, intitulada "Antropologia e História", o próprio Evans-Pritchard afirmava:

I would say that a term like "structure" can only be meaningful when used as an historical expression to denote a set of relations known to have endured over a considerable period of time. (1962: 55) 
A importância da história na própria noção de estrutura segundo Evans-Pritchard não poderia ser mais clara. E diante dos fatos recentes, as estruturas que revelou entre os Nuer parecem ter passado pelo teste que ele mesmo propunha: não só estrutura que permite compreender a história, mas estrutura que se revela tal ao perdurar na história. "History - lembra Evans-Pritchard mais adiante, citando Dumont - is the movement by which a society reveals itself as what it is" (: 56). Seis décadas após a publicação de Os Nuer, pode-se ainda reconhecê-los na análise de Evans-Pritchard.

\section{Epílogo}

As últimas notícias disponíveis quanto a essas negociações de paz no Sudão meridional datam de maio de 2000 (Acordo de Liliir). As notícias mais recentes do Sudão se referem à guerra entre o sul e o norte, na qual Dinka e Nuer, bem como os demais grupos "animistas e cristãos" do sul aparecem como "rebeldes", e têm contado com o apoio crescente dos Estados Unidos. No que diz respeito aos fatos relativos à paz no Sudão meridional que nos interessam aqui, de qualquer modo, só nos resta constatar que, graças a Evans-Pritchard, colocados diante de acontecimentos recentes, que afetam povos remotos de um país longínquo, somos capazes de compreender notícias que de outro modo pareceriam totalmente "exóticas", no pior dos sentidos. E que a compreensão das estruturas permite também ler a história. Ou ainda, retomando o próprio Evans-Pritchard, que estruturas de fato são aquelas que persistem.

\section{Notas}

1 Este artigo nasceu de uma pesquisa a respeito da situação atual dos Nuer, voltada para um curso de graduação cujo programa incluía a leitura integral da monografia clássica de Evans-Pritchard. A intenção era meramente responder perguntas 
como: “os Nuer ainda estão lá?”, "quantos são?” etc. As informações encontradas ultrapassaram muito nossa expectativa, sobretudo porque mostravam que, além dos Nuer estarem lá, e serem numerosos, é possível reconhecê-los na obra de Evans-Pritchard, e especialmente graças a ela. Agradeço aqui aos alunos, cuja reação entusiasmada me incentivou a redigir este artigo, e a José Guilherme C. Magnani, por conversas e sugestões.

2 O artigo do W ashington Post, intitulado "Sudanese tribes confront modern war", encontra-se em < http:/ /www.washingtonpost.com/wp-srv/inatl/galleries/ sudan/front.htm $>$.

3 Seriam aproximadamente 1.350.000 Dinka (Summer Institute of Linguistics,). Devido à proporção demográfica, todas as notícias se referem aos Dinka em primeiro lugar, o que de certo modo desloca a perspectiva "nuer-centrada" a que nós, leitores de Os Nuer, estamos habituados.

4 Os conflitos entre as populações do Sudão meridional e as populações árabes ao norte são bem mais antigos, sendo até mesmo mencionados, diversas vezes, pelo próprio Evans-Pritchard. Após a independência, tomam uma forma nova e, nesse sentido, pode-se dizer que uma outra guerra, agora contra um governo muçulmano, inicia em 1955, logo antes, portanto, da independência oficial. Essa guerra, de proporções e conseqüências devastadoras, que constitui a razão de ser dos esforços de pacificação no Sudão meridional, não será tratada neste artigo.

5 Compreende-se que os dados demográficos mais recentes datem de 1982.

6 A formulação de Evans-Pritchard é mais detalhada: "As soon as a man slays another he hastens to the home of a leopard-skin chief to cleanse bimself from the blood he has split and to seek sanctuary from the retaliation he has incurred. He may neither eat nor drink till the blood of the dead man has been let out of his body, for it is thought to pass into it some way, and to this end the chief makes one or two vertical incisions on his arm" (1940: 152).

7 Um dos indícios mais fortes do alcance e gravidade do conflito é a quantidade de crianças que compõem as tropas do SPLA. A 6 de setembro de 2001, uma nota de imprensa relatava a volta ao lar de "soldados" crianças: "Here in Sudan, some 10,000 children under 18 augment the ranks of the Southern People's Liberation 
Army (SPLA), which has been fighting a war of independence from the Islamist government for 18 years. But now, in a country where boys are initiated into manhood at age 11 - and are called to war as early as 8- some 3,500 child soldiers are getting a second chance at being youths." "'In Sudan, soldiers become children again", <http: SudanInfonet.tripod.com>).

8 Cf. William Lowrey; Dinka-Nuer Press Release \#2, 20/02/99.

9 Como veremos mais adiante, vários outros acordos de paz seriam firmados, sobretudo entre segmentos nuer, sem os quais a almejada paz no Sudão meridional não podia ser alcançada.

10 Em comunicado de 15 de junho de 1999, uma nota de imprensa do Sudanese Catholic Information Office (SCIO) informava que havia sido instalada, em Wunlit, uma dessas escolas. Até aquele momento, só havia na escola alunos dinka, "but the Nuer are most welcome as it is situated at a common border", obervava o Bispo de Rumbek SCIO-Sudan Monthly Report, 15/6/99.

11 Dinka-Nuer West Bank Peace Council (Yirol). Communication, 30/9/99.

12 O nível de detalhamento e precisão das informações desse item pode ser apreciado em passagens como: "The Dinka who went to raid Nuer were provoked by Nuer who came to raid cattle from Agar and which were then retrieved by SPLA soldiers who then returned to abduct the girl named Ayiep Manyiel Atembai which bad caused the raid of Nuer cattle by Dinka".

13 Diz o item: "In case of any marriage the father of the girl from the two parties should delegate a number of relatives to settle the marriage in order to collect the bride price in a group rather than a single person coming to collect the bride price and taking a different direction without reporting back to relatives who will later deny that the bride price has been received'. A presença dessa sugestão revela, ao mesmo tempo, a importância do pagamento do "preço da noiva" e a preocupação em evitar qualquer fonte de conflito. O Apêndice fornece os nomes de todas as pessoas que foram devolvidas, bem como daquelas ainda desaparecidas e das que quiseram permanecer onde estavam. Detalha, do mesmo modo, as negociações dos casamentos, com suas "conversas de gado" e relações de parentesco: "Nyieth Agontol from Agar Pakam section married the daughter of a Nuer with a bride price of 10 bead of cattle and they were received by Chief Kok Yar of Nuer", "The danghter of Cieny Buoth, named Akuac, has 
Revista de Antropologia, São Paulo, USP, 2001, v. 44 nº 2.

married a man named Malieny with the bride price of 40 cows having been paid", "The wife of Matueng Angong, a woman from Nuer, is now officially married with the payment of five cows by the family of the husband", "The danghter of Manyuel Bapal was staying with her maternal uncle, who is from Agar Dinka, and married an Agar man of the area. Chief Manyiel Lieny of Pakam is the uncle of the husband and has officially concluded the marriage with the bride price payment of 40 beard of cattle made to her parents in Nuer area" (Dinka-Nuer West Bank Peace Council (Yirol). Communication, 30/9/99).

14 Dinka-Nuer Press Release \#4, 13/03/99.

15 Dinka-Nuer Press-Release \#2, 20/02/99.

16 Tanto os comentários relativos a esse acordo quanto o texto do próprio acordo se encontram em notas de imprensa do New Sudan Council of Churches, e se encontram em $<$ http: //members.tripod.com/ $\sim$ SudanInfonet/ $>$. A conclusão de uma dessas notas louva a paz "across denominations", claramente reduzindo os segmentos a meros nomes diferentes. Note-se que Evans-Pritchard aponta como "one of the worst wars in Nuer history" aquela, então recente, ocorrida entre "the Gun and Mor moities of the Lou tribe", narrando em seguida outros enfrentamentos entre segmentos dos mesmos Lou (1940: 144).

17 Liliir Peace Conference. Covenant. 15/5/00.

18 Foram sobretudo autores franceses, como Lévi-Strauss e Dumont, que fizeram notar o quanto a noção de estrutura de Evans-Pritchard se afastava da de RadcliffeBrown. Em seu Prefácio à edição francesa de Os Nuer, Dumont fala numa "revolução", que o próprio Evans-Pritchard teria escolhido não sublinhar à época da publicação de Os Nuer, mas que seria declarada, com todas as suas conseqüências, na conferência “Social Anthropology, Past and Present”, em 1950, e que consumaria "le schisme avec Radcliffe-Brown" (: viii).

19 Publicada em 1949, que o próprio Evans-Pritchard aponta modestamente como "one of the few genuinely historical books written by an anthropologist de carrière" (1962: 58). 


\section{Bibliografia}

\section{DINKA-NUER WEST BANK PEACE AND RECONCILIATION} CONFERENCE

(Wunlit), Resolutions. <http://Sudaninfonet.tripod.com>, 10/3/1999.

DINKA-NUER WEST BANK PEACE COUNCIL

(Yirol), Communication. <http://Sudaninfonet.tripod.com>, 30/9/1999.

DUMONT, L.

1994

"Prefácio", in EVANS-PRITCHARD, E. E., Les Nuer, Paris, Gallimard.

EVANS-PRITCHARD, E. E.

1940 The Nuer. A description of the modes of livelihood and political institutions of a nilotic people, Nova Iorque/Oxford, Oxford University Press.

1951 Kinship and Marriage among the Nuer, Oxford, Clarendon Press.

1962 Essays in Social Anthropology, Londres, Faber and Faber.

IN SUDAN, SOLDIERS BECOME CHILDREN AGAIN.

$<$ http://Sudaninfonet.tripod. com>, 6/9/2001.

\section{LILIIR PEACE CONFERENCE}

Covenant. <http://Sudaninfonet.tripod.com>, 15/5/2000.

LOWREY, W. O.

"A Flicker of Hope in Sudan". <http://Sudaninfonet.tripod.com>, $15 / 5 / 2000$.

NSCC. NEW SUDAN COUNCIL OF CHURCHES.

Dinka-Nuer Reconciliation and Peace Workshop Communique. <http:/ /Sudaninfonet.tripod.com>, 15/6/1999.

Dinka-Nuer Press Releases. <http://Sudaninfonet.tripod.com>, \# 1 (9/2/1999), \# 2 (20/2/1999), \# 4 (13/3/1999); Press Release 12/11/1999. 
Revista de Antropologia, São Paulo, USP, 2001, v. 44 nº 2.

SCIO. SUDAN CATHOLIC INFORMATION OFFICE.

Sudan Monthly Reports. <http://Sudaninfonet. tripod.com>, 15/4/ 1998, 15/7/1999.

SUMMER INSTITUTE OF LINGUISTICS

<http://www.sil.org/ethnologue/>.

THE WASHINGTON POST

Sudanese Tribes confront modern war. <http://www.washingtonpost. com/wp-srv/inatl/galleries/sudan/ front.htm>, 7/7/1999.

WAAT LOU NUER COVENANT.

$<$ http://Sudaninfonet.tripod.com>, 6/11/1999. 
ABSTRACT: In March 2000, a Dinka-Nuer peace treaty was signed in southern Sudan. The civil war that had been going on for decades, as well as the resolutions adopted, described in the press and present in the documents, acquire full meaning when one considers Evans-Pritchard's deep analysis of Nuer politics. The nuer structures revealed in his works, having persisted for decades, pass the "test of history", and lead to a reconsideration of his contributions to the understanding of the inter-play between structure and history.

KEY-WORDS: Nuer, Dinka, Southern Sudan, Evans-Pritchard, structure, history.

Recebido em outubro de 2001. 